\title{
Bladder Recovery Patterns in Patients with Complete Cauda Equina Syndrome: A Single- Center Study
}

\author{
Ashok Pedabelle Reddy, Rajat Mahajan, Tarush Rustagi, Harvinder Singh Chhabra \\ Department of Spine Surgery, Indian Spinal Injuries Centre, New Delhi, India
}

\section{Study Design: Retrospective case series.}

Purpose: Cauda equina syndrome (CES) is associated with etiologies such as lumbar disc herniation (LDH) and lumbar canal stenosis (LCS). CES has a prevalence of $2 \%$ among patients with LDH and exhibits variable outcomes, even with early surgery. Few studies have explored the factors influencing the prognosis in terms of bladder function. Therefore, we aimed to assess the factors contributing to bladder recovery and propose a simplified bladder recovery classification.

Overview of Literature: Few reports have described the prognostic clinical factors for bladder recovery following CES. Moreover, limited data are available regarding a meaningful bladder recovery status classification useful in clinical settings.

Methods: A single-center retrospective study was conducted (April 2012 to April 2015). Patients with CES secondary to LDH or LCS were included. The retrieved data were evaluated for variables such as demographics, symptom duration, neurological symptoms, bladder symptoms, and surgery duration. The variable bladder function outcome during discharge and at follow-up was recorded. All subjects were followed up for at least 2 years. A simplified bladder recovery classification was proposed. Statistical analyses were performed to study the correlation between patient variables and bladder function outcome.

Results: Overall, 39 patients were included in the study. Majority of the subjects were males (79.8\%) with an average age of 44.4 years. CES secondary to LDH was most commonly seen (89.7\%). Perianal sensation (PAS) showed a significant correlation with neurological recovery. In the absence of PAS, bladder function did not recover. Voluntary anal contraction (VAC) was affected in all study subjects.

Conclusions: Intactness of PAS was the only significant prognostic variable. Decreased or absent VAC was the most sensitive diagnostic marker of CES. We also proposed a simplified bladder recovery classification for recovery prognosis.

Keywords: Cauda equina syndrome; Neurogenic urinary bladder; Lumbar disc; Lumbar stenosis

\section{Introduction}

Cauda equina syndrome (CES) is a clinical condition characterized by impaired bladder and bowel function or compromised sexual function caused by the compression of the cauda equina (sacral roots) [1]. Complete CES is defined by complete loss of bladder control and retention of urine with or without overflow incontinence [2]. CES can occur secondary to any condition resulting in the narrowing of the spinal canal, such as lumbar disc herniation (LDH),

Received Feb 28, 2018; Revised Apr 10, 2018; Accepted Apr 21, 2018

Corresponding author: Tarush Rustagi

Department of Spine Surgery, Indian Spinal Injuries Centre, Vasant Kunj, New Delhi, Delhi-110070, India

Tel: +91-8826383705, Fax: ++91-1127318797, E-mail: Spineclinicdelhi@gmail.com 
lumbar canal stenosis (LCS), lumbar spine fracture, tumors, infections, and spondylolisthesis. LDH is the most common cause of CES and accounts for $43 \%$ of all cases [3].

Bladder involvement and recovery are important aspects of CES that need to be discussed with the patient $[4,5]$. Limited data are available to be used for the identification of the clinical factors that may help prognosticate bladder recovery. Our study aim was to assess the cohort of patients with CES, identify the factors influencing bladder recovery, and propose a simple bladder recovery classification.

\section{Materials and Methods}

The study was approved by the Institutional Review Board of Indian Spinal Inquires Centre (IRB approval no., ISIC/ $\mathrm{RP} / 2018 / 095)$. IRB waived the requirement for informed consent. We conducted a retrospective case series at a single tertiary center. All the case files were retrieved from the institutional database. The study cohort included all patients diagnosed with CES secondary to LDH and LCS (involving the lumbar two to sacrum) operated from April 2012 to April 2015. CES was identified and defined by Fraser et al. [1]. The clinical assessment included recording the perianal sensation (PAS; patient reporting as nor$\mathrm{mal} /$ decreased/absent PAS) or voluntary anal contraction (VAC; recorded as normal/weak/absent tone). The time of CES onset was defined as the time when the patient was first identified to experience bladder problems and was classified as $<48$ hours or $>48$ hours.

We created three categories of bladder recovery pattern based on the clinical situation and post-void ultrasonography performed every 6 months (follow-up), and the variables were studied. We defined bladder recovery as 'complete' if the patient did not exhibit any residual bladder symptoms and 'partial' if the patient required to strain but did not require clean intermittent catheterization and had a residual urine volume $<100 \mathrm{~mL}$. Those who required intermittent catheterization or had a residual volume $>100$
$\mathrm{mL}$ were considered to have 'no recovery' (Table 1).

Variables, including demographics, and magnetic resonance imaging (MRI) results were recorded. Data were entered in a Microsoft Excel sheet (Microsoft Corp., Redmond, WA, USA), and statistical analyses were performed using IBM SPSS software ver. 20.0 (IBM Corp., Armonk, NY, USA). Fisher exact test and multiple logistic regression analyses were performed to evaluate the significance of various variables in the outcomes.

\section{Results}

\section{Demographic data}

Overall, 39 patients were included in the study (Fig. 1). The average age of the subjects was 44.4 years (range, 2072 years), and the majority were males (31 males and eight females). The average follow-up duration was 41 months (range, 24-60 months). Among the 39 cases, 35 (89.7\%)

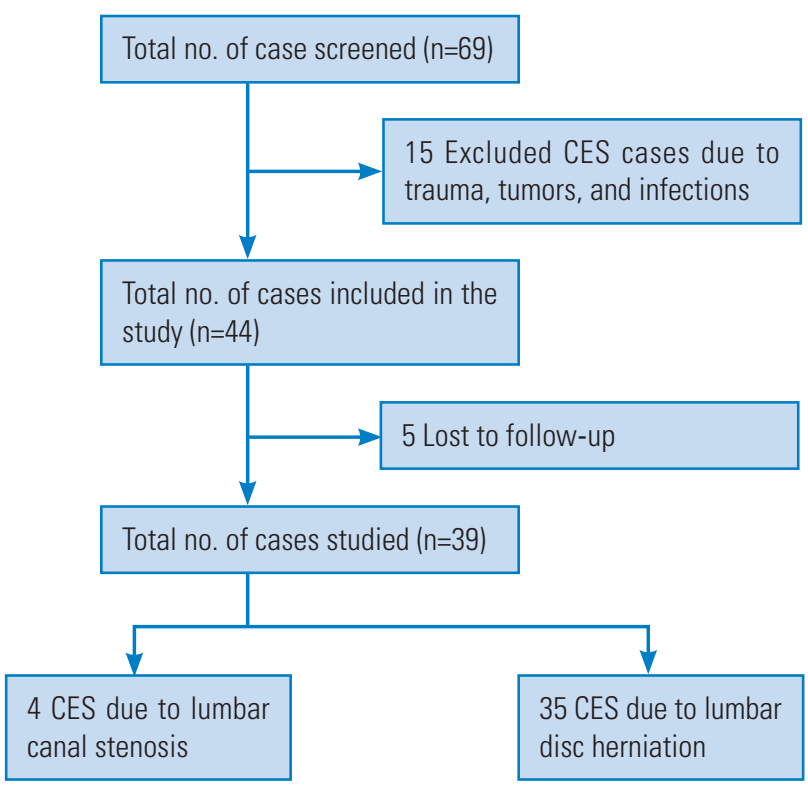

Fig. 1. Flowchart showing patient selection. CES, cauda equina syndrome.

Table 1. Categories of bladder recovery

\section{Type of bladder recovery Residual bladder symptoms}

Clean intermittent catheterization requirement

Post void urine volume

$\begin{array}{ll}\text { No } & \text { Minimum } \\ \text { No/occasionally } & <100 \mathrm{~mL} \\ \text { Yes } & -\end{array}$


had LDH, whereas four had LCS as the cause of CES. During surgery, 28 of the 35 patients in the LDH group underwent laminectomy with discectomy of the involved level, and seven had decompression with posterior fusion (two had posterolateral fusion and five had interbody fusion). In the LCS group, two patients had decompression with interbody fusion and two patients underwent laminectomy.
Nine patients (23\%) had the onset of bladder symptoms in $<48$ hours; seven of them showed complete or partial recovery. In the other group comprising patients who developed bladder symptoms after 48 hours, 25 of the 30 patients showed complete or partial bladder recovery (Table 2).

At presentation, PAS was labeled as 'present,' 'decreased,' or 'absent' in 19 (48.71\%), 17 (43.58\%), and three patients (7.69\%), respectively. VAC was reported as 'weak' or 'absent'

Table 2. Association of various factors with the bladder recovery pattern $(\mathrm{N}=39)$

\begin{tabular}{|c|c|c|c|c|c|}
\hline Variable & No recovery & Partial recovery & Complete recovery & Total & $p$-value \\
\hline Age group (yr) & & & & & 0.496 \\
\hline $21-40$ & 2 & 7 & 6 & 15 & \\
\hline $41-60$ & 4 & 7 & 9 & 20 & \\
\hline$>61$ & 1 & 3 & 0 & 4 & \\
\hline Sex & & & & & 0.268 \\
\hline Female & 0 & 5 & 3 & $8(20.5)$ & \\
\hline Male & 7 & 12 & 12 & $31(79.5)$ & \\
\hline \multicolumn{6}{|l|}{ Onset of symptoms } \\
\hline$<48 \mathrm{hr}(\mathrm{N}=9)$ & 2 & 3 & 4 & 9 & 0.69 \\
\hline$>48 \mathrm{hr}(\mathrm{N}=30)$ & 5 & 14 & 11 & 30 & 0.69 \\
\hline Diagnosis & & & & & 0.21 \\
\hline Lumbar disc herniation & 5 & 16 & 14 & 35 & \\
\hline Lumbar canal stenosis & 2 & 1 & 1 & 4 & \\
\hline Level & & & & & 0.48 \\
\hline $\mathrm{L} 2-\mathrm{L} 3$ & 0 & 1 & 0 & $1(2.56)$ & \\
\hline L3-L4 & 2 & 1 & 1 & $4(10.25)$ & \\
\hline L4-L5 & 4 & 7 & 7 & $18(46.15)$ & \\
\hline L5-S1 & 1 & 8 & 7 & 16 (41.02) & \\
\hline Total outcome & $7(17.8)$ & 17 (43.6) & 15 (38.6) & $39(100.0)$ & \\
\hline
\end{tabular}

Values are presented as number or number (\%).

Table 3. Clinical examination and recovery pattern

\begin{tabular}{|c|c|c|c|c|}
\hline Examination and recovery pattern & No recovery & Partial recovery & Complete recovery & Total \\
\hline \multicolumn{5}{|l|}{ Perianal sensation } \\
\hline Absent & $3(100.0)$ & 0 & 0 & $3(7.69)$ \\
\hline Decreased & $2(11.75)$ & $13(76.5)$ & $2(11.75)$ & $17(43.58)$ \\
\hline Present & $2(10.6)$ & $4(21.0)$ & $13(68.4)$ & $19(48.71)$ \\
\hline \multicolumn{5}{|l|}{ Voluntary anal contraction } \\
\hline Absent & 4 & 5 & $4(30.76)$ & $13(33.33)$ \\
\hline Weak & 3 & 12 & $11(42.3)$ & $26(66.67)$ \\
\hline Present & 0 & 0 & 0 & 0 \\
\hline Total outcome & $7(17.8)$ & $17(43.6)$ & $15(38.6)$ & $39(100.0)$ \\
\hline
\end{tabular}

Values are presented as number (\%) or number. 
Table 4. Association of PAS and VAC with the bladder recovery pattern

\begin{tabular}{lcccc} 
Clinical examination & $\begin{array}{c}\text { PAS present/reduced } \\
\text { and VAC weak (N=26) }\end{array}$ & $\begin{array}{c}\text { PAS present or reduced } \\
\text { with VAC absent (N=10) }\end{array}$ & $\begin{array}{c}\text { PAS absent and VAC } \\
\text { absent (N=3) }\end{array}$ & 0 \\
Complete bladder recovery pattern & 11 & 4 & 0 & 0.001 \\
Partial bladder recovery & 12 & 5 & 3 \\
\hline No bladder recovery & 3 & 1 & 0 \\
\hline
\end{tabular}

PAS, perianal sensation; VAC, voluntary anal contraction.

in $26(66.66 \%)$ and 13 patients (33.33\%), respectively (Table 3). No patient diagnosed with CES had normal VAC.

MRI suggested L4-L5 level as the most frequent compression site $(\mathrm{n}=18,46.15 \%)$, followed by L5-S1 in 16 patients (41.02\%), L3-L4 in four patients (10.25\%), and L2L3 in one patient $(2.56 \%)$ (Table 2 ). Overall, the outcome of bladder recovery pattern was determined as 'complete' in 15, 'partial' in 17 patients, and 'no recovery' in seven patients $(17.8 \%)$.

\section{Correlation of various variables with bladder recov- ery outcome}

1) Age, sex, and diagnosis

Majority of the patients were 41-60 years old $(n=20)$. The age of presentation and sex did not affect the bladder recovery outcome ( $p=0.496$ and 0.268 , respectively). Majority of the patients developed CES secondary to LDH $(n=35)$; most patients with LDH $(30 / 35)$ showed partial or complete recovery, whereas $50 \%$ of patients with LCS showed no recovery. However, the difference was not significant $(p=0.21)$.

2) Perianal sensation and voluntary anal contraction

As seen in Table 4, 23 of the 26 patients with complete or partial bladder recovery pattern had reported PAS as normal or reduced, and VAC was found to be weak. This was observed in nine of the 10 patients when the PAS was reported as normal or weak, and VAC was found to be absent. No bladder recovery was observed for patients with no PAS or VAC; this association was statistically significant $(p<0.001)$.

\section{3) Lumbar level of involvement}

As only one patient had L2-L3 involvement, the statistical correlation could not be tested. Among the other three levels, lower level stenosis showed a non-significant, but better, recovery pattern $(p=0.48)$.
4) Duration of symptoms prior to surgical decompression Among the two groups ( $<48$ hours and $>48$ hours), there was no difference in the outcomes $(p=0.658)$.

\section{Discussion}

In the current case series, we proposed a simple classification for bladder recovery pattern following CES. We found that patients with the presence or reduction of PAS were significantly more likely to show a complete or partial recovery pattern, irrespective of VAC. We also found that altered VAC had $100 \%$ sensitivity for the diagnosis of CES.

The purpose of classifying bladder recovery pattern was to establish the prognosis, predict the need for self-catheterization, and determine whether the post-void urine volume would be detrimental to the upper urinary tract. The cutoff point indicating significant post-void urine volume was unclear. We considered the post-void volume as $<100 \mathrm{~mL}$ based on the general recommendation of the urologist who considered any residue $>100 \mathrm{~mL}$ as abnormal [6].

CES can have diverse phenotypes; the most common symptom is difficulty in urination (bladder symptoms), followed by bowel incontinence, constipation, and sexual dysfunction [4]. Bladder symptoms can either be complete (CES-R) or incomplete (CES-I) [5]. CES-R patients are those with complete loss of voluntary bladder control along with either acute retention or overflow incontinence. CES-I patients present with vague symptoms such as sensation of incomplete voiding, urgency, poor urinary stream, and urinary straining [5]. In their study, Gardner et al. [5] noted that 50\%-70\% of patients had CES-R with poor prognosis, whereas CES-I patients accounted for $30 \%-40 \%$ of the cases and had a good prognosis [5]. Our series included both types of patients, and we did not assess the completeness of CES in our study.

Balasubramanian et al. [7] noted that the only signifi- 
cant finding associated with CES is saddle anesthesia. It is caused by the compression of the S2, S3, and S4 roots and can be evaluated clinically by checking PAS; it can be graded as normal, absent, or decreased. We noticed that PAS was affected only in $51.3 \%$ of the patients.

It may be debatable as to how PAS was reported as 'normal' in $49.7 \%$ cases of CES where saddle anesthesia was a hallmark; it is difficult to explain this finding. We believe that PAS is a subjective finding, and the data were recorded as reported by the patient; therefore, there is a potential for bias. The patients who reported normal PAS may have actually experienced reduced sensation that they were unable to categorically report. However, we found that the mere presence of PAS (irrespective of the degree) was significantly related to improved bladder recovery.

VAC is affected in CES and is decreased or absent in patients with CES. A range of $7.6 \%-52 \%$ has been reported in the literature for the association of VAC with CES $[8,9]$. We found that VAC was either absent or weak in all the patients. This was an objective finding; therefore, it is less likely to be a false positive. The presence of VAC abnormality had $100 \%$ sensitivity for establishing a diagnosis.

In the current study, bladder function was 'complete, 'partial,' or 'no recovery' in $38.6 \%, 43.6 \%$, and $17.8 \%$ of the patients, respectively. Our findings were similar to those of Beculic et al. [10] who reported that 36\%, 36\%, and $28 \%$ of the patients had normal, partial retention, and complete retention of bladder function, respectively, at the final follow-up.

Beculic et al. [10] reported L4-L5 as the most commonly involved level in $\mathrm{LDH}$, similar to our finding. In their meta-analysis, Ahn et al. [4] reported L1-L2 as the most frequently involved level, followed by the L3-L4 level. We excluded the L1-L2 level because periconal injuries (conus medullaris syndrome) have a different clinical course and could have confounded the results. In our series, a low compression level was better for bladder recovery. This may have been secondary to the partial preservation of the sacral roots.

The surgery duration in CES from the time of symptom onset has been extensively researched by several authors, and early surgery is recommended, when feasible $[3,4,10]$. Most patients presented after 48 hours of symptom onset; therefore, we were unable to establish any association with the time of presentation in terms of bladder recovery. Our findings were similar to those reported by Korse et al. $[3,11]$ who found no significant difference in the outcomes of the early and late surgical decompression groups.

All the study subjects underwent decompression with or without fusion. We did not assess the correlation between bladder recovery and surgery type. We believe that the primary objective of surgery was adequate decompression, and fusion was based on any obvious or impending instability.

One important limitation of our study was that we did not assess the CES type in terms of whether it was complete or incomplete. This may have influenced the overall outcome. Retrospectively, if the patients were classified as per the 'Shi classification', three who showed no recovery would fall under the 'late' category, whereas the remaining would fall under the 'early' or 'late' categories [12].

Investigators often hesitate to perform PAS and VAC because of their invasiveness and patient discomfort [13]. We believe that these tests must be discussed with the patient in detail, including their clinical utility to determine the postoperative bladder recovery status.

\section{Conclusions}

We described a simple and reproducible bladder recovery pattern classification in our series. VAC proved to be extremely sensitive for diagnosing CES. The presence of PAS was the only significant prognostic variable for bladder recovery.

\section{Conflict of Interest}

No potential conflict of interest relevant to this article was reported.

\section{References}

1. Fraser S, Roberts L, Murphy E. Cauda equina syndrome: a literature review of its definition and clinical presentation. Arch Phys Med Rehabil 2009;90:19648.

2. Gleave JR, Macfarlane R. Cauda equina syndrome: what is the relationship between timing of surgery and outcome? Br J Neurosurg 2002;16:325-8.

3. Korse NS, Jacobs WC, Elzevier HW, VleggeertLankamp CL. Complaints of micturition, defecation and sexual function in cauda equina syndrome due to lumbar disk herniation: a systematic review. Eur 
Spine J 2013;22:1019-29.

4. Ahn UM, Ahn NU, Buchowski JM, Garrett ES, Sieber AN, Kostuik JP. Cauda equina syndrome secondary to lumbar disc herniation: a meta-analysis of surgical outcomes. Spine (Phila Pa 1976) 2000;25:1515-22.

5. Gardner A, Gardner E, Morley T. Cauda equina syndrome: a review of the current clinical and medicolegal position. Eur Spine J 2011;20:690-7.

6. Kelly CE. Evaluation of voiding dysfunction and measurement of bladder volume. Rev Urol 2004;6 Suppl 1:S32-7.

7. Balasubramanian K, Kalsi P, Greenough CG, Kuskoor Seetharam MP. Reliability of clinical assessment in diagnosing cauda equina syndrome. Br J Neurosurg 2010;24:383-6.

8. Ahad A, Elsayed M, Tohid H. The accuracy of clinical symptoms in detecting cauda equina syndrome in patients undergoing acute MRI of the spine. Neuroradiol J 2015;28:438-42.
9. Gooding BW, Higgins MA, Calthorpe DA. Does rectal examination have any value in the clinical diagnosis of cauda equina syndrome? Br J Neurosurg 2013;27:156-9.

10. Beculic H, Skomorac R, Jusic A, et al. Impact of timing on surgical outcome in patients with cauda equina syndrome caused by lumbar disc herniation. Med Glas (Zenica) 2016;13:136-41.

11. Korse NS, Pijpers JA, van Zwet E, Elzevier HW, Vleggeert-Lankamp CLA. Cauda Equina Syndrome: presentation, outcome, and predictors with focus on micturition, defecation, and sexual dysfunction. Eur Spine J 2017;26:894-904.

12. Ma B, Wu H, Jia LS, Yuan W, Shi GD, Shi JG. Cauda equina syndrome: a review of clinical progress. Chin Med J (Engl) 2009;122:1214-22.

13. Cohen SP, Argoff CE, Carragee EJ. Management of low back pain. BMJ 2008;337:a2718. 